Mathematical Medicine and Biology (2017) 34, 59-75

doi:10.1093/imammb/dqv037

Advance Access publication on November 20, 2015

\title{
A non-local model for cancer stem cells and the tumour growth paradox
}

\author{
I. BORSI \\ TEA Sistemi, Via Ponte a Piglieri, 8, 50122 Pisa, Italy \\ iacopo.borsi@tea-group.com \\ A. FASANO \\ Dipartimento di Matematica e Informatica 'U. Dini', Università degli Studi di Firenze, Viale Morgagni \\ 67/A, 50134 Firenze, Italy and FIAB. SpA, via Passerini 2, 4, 6 - 50039 Vicchio - Firenze, Italiy \\ fasano@math.unifi.ita.fasano@fiab.it \\ M. PRIMICERIO \\ Dipartimento di Matematica e Informatica 'U. Dini', Università degli Studi di Firenze, Viale Morgagni \\ 67/A, 50134 Firenze, Italy \\ primicer@math.unifi.it \\ AND \\ T. HILLEN* \\ Centre for Mathematical Biology, Mathematical and Statistical Sciences, University of Alberta, \\ Edmonton, AB, Canada T6G $2 G 1$ \\ *Corresponding author: thillen@ualberta.ca
}

[Received on 24 October 2014; revised on 20 May 2015; accepted on 1 October 2015]

\begin{abstract}
The tumour growth paradox refers to the observation that incomplete treatment of cancers can enhance their growth. As shown here and elsewhere, the existence of cancer stem cells (CSCs) can explain this effect. CSC are less sensitive to treatments, hence any stress applied to the tumour selects for CSC, thereby increasing the fitness of the tumour. In this paper, we use a mathematical model to understand the role of CSC in the progression of cancer. Our model is a rather general system of integro-differential equations for tumour growth and tumour spread. Such a model has never been analysed, and we prove results on local and global existence of solutions, their uniqueness and their boundedness. We show numerically that this model exhibits the tumour growth paradox for all parameters tested. This effect becomes more relevant for small renewal rate of the CSC.
\end{abstract}

Keywords: cancer stem cells; integro-differential equations.

\section{Introduction}

Tumour stem cells or cancer stem cells (CSC) have been identified in many cancers, including carcinomas of the breast, brain, colon, prostate, pancreas, ovary, as well as in sarcomas and in leukaemia (Dittmar \& Zänker, 2013). Stem cells in general are pluripotent and they generate cells of various cell lineages. In the haematopoietic system, for example, the progenitor cells are often classified as multipotent progenitors who give rise to a lineage of transient, transient amplifying and differentiated cells. In cancer these differentiation stages are more diffuse and a clear distinction between these stages is often not possible. Hence, to investigate the role of stem cells, we combine all non-stem cancer cells 
into one group called cancer cells (CCs). While it is not always easy to identify CSCs, it is widely accepted that they are instrumental in tumour progression and control. In fact, the control or eradication of CSC has become a focus for treatment design (Dingli \& Michor, 2006).

In this article, we develop and analyse a mathematical model for CSC and CC which consists of a non-linear coupled system of integro-differential equations (iDEs). Existence results for this coupled system are not readily available, hence we prove mathematical properties on positivity, boundedness, existence and uniqueness. We show numerically that this iDE model supports a tumour growth paradox, i.e. the fact that a tumour with larger death rate for CC might grow bigger than a tumour with lower $\mathrm{CC}$ death rate. We then analyse the sensitivity of the tumour growth paradox on the spatial spread rates of the tumour cells. We find that the tumour growth paradox arises for all tested parameter values. It is more pronounced in cases of low mobility of the CSC and a low renewal rate for CSC. Finally, we consider an example of incomplete radiation treatment, where the tumour after radiation grows larger than it would have grown if left untouched-the tumour growth paradox at work.

\subsection{Modelling of CSCs}

Mathematical modelling is an established method to help to understand complex biological systems. Related to tumour stem cells several models have been developed recently (Dingli \& Michor, 2006; Ganguli \& Puri, 2006; Sole et al., 2008; Beretta et al., 2012; Stiehl \& Marciniak-Czochra, 2012; Sun \& Komarova, 2012; Hillen et al., 2013). Aspects of CSCs that are of importance to cancer progression, including repopulation, treatment resistance, stem cell differentiation, positive and negative feedback loops, de-differentiation mechanisms, competition for oxygen and nutrients and spatial arrangements. Mathematical modelling of all these aspects in one big model is certainly possible, but its potential use is limited. The more detail we include, the more specific assumptions and parameter values are needed. However, mathematical modelling has the advantage that sub-models can be studied on their own, thereby allowing us to focus on one or two aspects at a time, which is often impossible in vivo. In this paper, we focus on the aspect of spatial propagation and spatial crowding by CSCs and non-stem CCs.

In this context, Enderling et al. (2009) developed an individual-based cellular automaton model, where individual cells are described by elements of a square grid. Each grid point can be either a CSC or a CC or empty. Cells are able to divide if a free grid cell is available near by, otherwise cells become dormant (quiescent). Enderling et al. study the effect of spatial inhibition on the simulated tumour. If the death rate for the CC is low, then CC quickly surround CSC, who in turn lose free space for replication. Hence they turn quiescent until the whole tumour stops growing (or grows very slowly). If, however, the death rate for the CC compartment is increased, for example due to treatment, then CSC find open space to grow into. They produce more CSC through occasional symmetric divisions and as a result the tumour becomes bigger. The effect that increased CC death can lead to a larger tumour has been termed the tumour growth paradox. In fact, it is commonly observed in clinical practice that incomplete treatment can lead to an increased tumour burden after treatment. Enderling et al. (2009) point out that their result requires some movability of CSC, otherwise the tumour growth paradox would not arise.

Hillen et al. (2013) recast the dynamics of the cellular automaton model of Enderling et al. (2009) as a system of integro-partial differential equations (iPDEs) for continuous population densities of CSC and CC. While the system was developed in Hillen et al. it was not analysed there. Instead, Hillen et al. simplified the iDE model into a (spatially homogeneous) system of ordinary differential equations (ODEs). For this, ODE system they used geometric singular perturbation theory (Hek, 2010) to mathematically prove the existence of a tumour growth paradox. The analysis of the full iPDE model for 
CSCs was left open and this is the topic of a recent paper by Maddalena (2014) and the present paper. We discuss Maddalena's results later in Section 1.2 in Remark (4).

In Section 1.2, we will motivate the iDE model for CSC and CC from biological principles. In Section 2, we will present a detailed mathematical analysis of the above model. Under rather weak assumptions, we can show that continuous solutions exist, they are unique and they depend continuously on the initial conditions. The model contains a crowding term $F(p)$ with crowding capacity 1 and we can prove that solutions stay bounded between 0 and 1 .

In Section 3, we showcase some numerical experiments of the above model. We show clearly that the tumour growth paradox arises, i.e. a tumour with a larger death rate for $\mathrm{CC}$ will outgrow a tumour with a lower death rate. The reason is that a larger death rate for $\mathrm{CC}$ leads to a selective advantage for the CSC. Hence, a tumour with larger death rate for CC is CSC dominated, while a tumour with lower death rate is CC dominated. We study the sensitivity of the tumour growth paradox on the model parameters and we find that the tumour growth paradox is more pronounced for slow moving CSC and in cases where the CSC renewal rate is small. Larger spread of cells leads to a more homogeneous distribution and to less effect of the tumour growth paradox.

Additionally, we show some qualitative simulations for incomplete treatment and we find scenarios where the tumour after treatment will grow bigger than the corresponding untreated tumour.

This is, of course, not the final answer and the above model shows many more interesting properties in both ways, mathematically as well as for the application. In Section 4, we will discuss the relevance of our findings, and possible future avenues of investigation.

\subsection{The iDE model for CSC and CC}

To motivate our iDE model, we first recall the ODE model of Hillen et al. (2013)

$$
\begin{aligned}
& \dot{u}=\delta \gamma F(p) u, \\
& \dot{v}=(1-\delta) \gamma F(p) u+\rho F(p) v-\alpha v,
\end{aligned}
$$

with $p=u+v$, where $u(t), v(t)$ denote time-dependent densities of CSC and CC, respectively. The parameter $\gamma>0$ denotes the average mitosis rate for CSC, while $\rho>0$ describes the mitosis rate for CC; $\alpha>0$ denotes the death rate of the CC cells. Death can be related to natural death or treatment induced cell death. The parameter $\delta>0$ describes the average fraction of CSC in the progeny of a CSC. There is no death term in the CSC compartment in this model, since we assume that CSC are immortal and also are less sensitive to treatment. It is an extreme case, and a small death rate for CSC could be incorporated if needed. The $F(p)$ term describes competition for space. $F(p)$ is a monotonically decreasing function describing the inhibitory effect of a cell density $p$. If $p$ exceeds a given crowding threshold $p^{*}$, then $F(p)=0$ for all $p \geqslant p^{*}$. Here we normalize $p^{*}=1$.

Hillen et al. proved in Hillen et al. (2013) that the above model (1.1) shows a tumour growth paradox, which, for this and more general cases, we define as follows.

Definition 1.1 Let $p_{\alpha}(t)$ denote the total tumour size at time $t>0$, where $\alpha \geqslant 0$ denotes the death rate of non-stem CCs. The model exhibits a tumour growth paradox if there exist death rates $\alpha_{1}<\alpha_{2}$ and a time interval $\left(t_{1}, t_{2}\right), t_{2}>t_{1}>0$, such that $p_{\alpha_{1}}(0)=p_{\alpha_{2}}(0)$ and

$$
p_{\alpha_{1}}(t)<p_{\alpha_{2}}(t) \quad \text { for all } t \in\left(t_{1}, t_{2}\right) \text {. }
$$


Hillen et al. (2013) showed that (1.1) always has at least two steady states $(0,0)$ and $(1,0)$ and a third steady state $\left(0, v_{0}\right)$ if the equation $F\left(v_{0}\right)=\alpha$ has a solution. The simplex $S:=\{u \geqslant 0, v \geqslant 0, u+v \leqslant 1\}$ is positively invariant and the only global attractor in $S$ is $(1,0)$. Hillen et al. (2013) used methods from geometric singular perturbation theory (Hek, 2010) and showed that for small $\delta>0$ there exists a slow manifold inside $S$ which attracts each orbit. The slow manifolds depend monotonically on $\alpha$ in such a way that the population with the larger $\alpha$ value does grow faster hence implying the tumour growth paradox. Enderling et al. (2009) argued that in their cellular automaton model the tumour growth paradox needed some form of spatial random movement. Hence, in this paper, we include spatial redistributions expressed through non-local integral terms to see if spatial movement would enhance, preserve or remove the tumour growth paradox when compared with (1.1).

To make this model spatially dependent, we use the general framework of a birth-jump process as introduced recently (Hillen et al., 2014). We describe spatial redistribution by an integral kernel $k(x, y, p(x, t))$. In our spatial model, we assume that at mitosis one daughter cell can take the location of the mother cell, while the other daughter cell is transported to another location. This can be nearby, depending on the kernel $k(x, y, p(x, t))$. The kernel $k$ is a transitional probability density in the sense that $k(x, y, p(x, t)) \Delta^{n} x$ is the probability that a daughter cell which is released at $y$ settles in an $n$-dimensional volume element at $x$ with side length $\Delta x$ per unit of time. This probability depends on the local density $p(x, t)$ to describe the volume filling effect. We choose

$$
k(x, y, p(x, t))=K(x, y) F(p(x, t))
$$

to separate redistribution $K(x, y)$ and the crowding effect $F(p)$. The spatial model reads:

$$
\begin{aligned}
& u_{t}(\mathbf{x}, t)=\delta \gamma \int_{\Omega} k(\mathbf{x}, \mathbf{y}, p(\mathbf{x}, t)) u(\mathbf{y}, t) \mathrm{d} \mathbf{y}, \\
& v_{t}(\mathbf{x}, t)=(1-\delta) \gamma \int_{\Omega} k(\mathbf{x}, \mathbf{y}, p(\mathbf{x}, t)) u(\mathbf{y}, t) \mathrm{d} \mathbf{y}+\rho \int_{\Omega} k(\mathbf{x}, \mathbf{y}, p(\mathbf{x}, t)) v(\mathbf{y}, t) \mathrm{d} \mathbf{y}-\alpha v(\mathbf{x}, t),
\end{aligned}
$$

where

$$
p(\mathbf{x}, t)=u(\mathbf{x}, t)+v(\mathbf{x}, t) .
$$

The spatial domain is denoted as $\Omega$. The functions $u(\mathbf{x}, t)$ and $v(\mathbf{x}, t)$ represent the densities of the CSCs and of the non-stem CCs, respectively. The parameters $\gamma>0$ and $\rho>0$ are the replication rates of the two families of cells; $\delta, 0<\delta<1$, is the average fraction of CSC in the progeny of a CSC; $\alpha$ is the mortality rate of the differentiated cells, and $k(\mathbf{x}, \mathbf{y}, p(\mathbf{x}, t))$ is the probability density that a cell located at $\mathbf{y}$ generates a cell at $\mathbf{x}$. We assume $k(\mathbf{x}, \mathbf{y}, p(\mathbf{x}, t))=K(\mathbf{x}, \mathbf{y}) F(p(\mathbf{x}, t))$, where

(A.1) $F(p)$ ranges in $[0,1]$ and is a non-negative non-increasing Lipschitz continuous function such that $F(0)=1, F(1)=0, F(p)>0$ for any $p$ in $(0,1)$ and $F(p)=0$ for $p>1$.

(A.2) $K \geqslant 0, K \in C(\bar{\Omega}, \bar{\Omega})$, and $\int_{\Omega} K(\mathbf{x}, \mathbf{y}) d \mathbf{y} \leqslant 1$.

\section{REMARKS}

(1) Note that system (1.2), (1.3) is not equipped with any boundary conditions. Since it is an iDE, boundary conditions are implemented by the use of the redistribution kernel $K$. For example, Dirichlet boundary conditions on a bounded domain $\Omega$ correspond to kernels that might have 
support outside of the domain $\Omega$. Then particles moving out of the domain will be lost permanently. Homogeneous no-flux conditions can be employed by requiring that redistribution always happens inside $\Omega$, i.e.

$$
\int_{\Omega} K(\mathbf{x}, \mathbf{y}) \mathrm{d} \mathbf{x}=1 \quad \text { for all } \mathbf{y} \in \Omega
$$

(2) Model (1.2), (1.3) falls into the class of birth-jump processes, which were introduced recently in Hillen et al. (2014). In a birth-jump process, the population growth and population spread are no longer independent processes. On the contrary, it is assumed that newly generated individuals are redistributed instantly. It should be noted that this choice does include standard diffusion terms as special cases. For example, if the kernel has the form $K(|\mathbf{x}-\mathbf{y}|)$ and if $K$ is highly concentrated, then a diffusion approximation of the integral can be performed. In fact, this will be done in a forthcoming paper, where we study invasion waves for a reaction-diffusion version of the above model.

(3) An advantage of the formulation of the above redistribution kernels is the fact that the quality and local occupancy of the target site can be directly included into the model. If the habitat is inhomogeneous and includes possible uninhabitable patches, then this can be expressed through the choice of $K(\mathbf{x}, \mathbf{y})$. In the context of cancer metastasis, the existence of niches for metastasis is discussed. Niches are favourable environments, where daughter tumours can form (Hanahan \& Weinberg, 2011), and this can be expressed through the kernel $K(\mathbf{x}, \mathbf{y})$.

(4) In the original iPDE of Hillen et al. (2013), the model equations (1.2), (1.3) also contain diffusion terms. If we abbreviate the integral terms as a $\star$ product $k \star u=\int_{\Omega} k(x, y, p(x, t)) u(y, t) \mathrm{d} y$, then the corresponding model is

$$
\begin{aligned}
& u_{t}=d_{u} \Delta u+\delta \gamma k \star u, \\
& v_{t}=d_{v} \Delta v+(1-\delta) \gamma k \star u+\rho k \star v-\alpha v,
\end{aligned}
$$

where $\Omega$ is a smooth bounded domain. Maddalena (2014) studies this model under von Neumann boundary conditions of the form

$$
\frac{\partial u}{\partial n}=\frac{\partial v}{\partial n}=0
$$

where $n$ denotes the outer normal on $\partial \Omega$. Based on general assumptions on the kernel $k$, the integral operators are compact operators on the Banach space $H^{2}(\Omega)$. The system (1.6) appears as heat equation with a compact and Lipschitz continuous perturbation. The solution theory for perturbed parabolic problems is available and Maddalena (2014) uses these methods to show results on existence, uniqueness, smoothness and invariant sets. Maddalena did not discuss how the homogeneous von Neumann boundary conditions (1.7) relate to possible boundary flux from the integral terms. It would be an interesting topic for future research to investigate the boundary conditions coming from the integral terms in relation to the classical conditions stemming from the diffusion terms. Since in our model (1.2), (1.3) we have no diffusion terms, we focus on the integral conditions such as (1.5). In our case, we study $d_{u}=d_{v}=0$. Then the leading-order terms are the integral terms. The regularity theory for uniform parabolic equations is no longer available and we find it necessary to develop a full solution theory here. 


\section{Existence, uniqueness and boundedness}

The above equations (1.2), (1.3) form a non-linear iDE system. A general existence theory for these type of systems has not yet been developed, hence we present a full solution theory here. We use a fixed-point argument to find unique continuous solutions. First, we derive some preliminary estimates, which also show continuous dependence of solutions on the initial conditions and uniqueness. We refine our a priori estimates to show that solutions stay in the interval $[0,1]$ for all time, if the initial conditions were in this interval. Finally, in Section 2.2 we construct a contraction mapping whose fixed point is a solution of (1.2), (1.3).

We assume that the initial conditions,

$$
u(\mathbf{x}, 0)=u_{0}(\mathbf{x}), \quad v(\mathbf{x}, 0)=v_{0}(\mathbf{x}), \quad \mathbf{x} \in \Omega,
$$

satisfy the following assumptions

(B.1) $u_{0}(\mathbf{x}), v_{0}(\mathbf{x}) \in C(\bar{\Omega})$,

(B.2) $u_{0} \geqslant 0, v_{0} \geqslant 0, u_{0}+v_{0} \leqslant 1$.

The existence problem of solutions for (1.2), (1.3) can be stated as:

Problem $(P)$. Find a pair $u(\mathbf{x}, t), v(\mathbf{x}, t)$ such that, for any $T>0$,

- $u, v \in C(\bar{\Omega} \times[0, T])$;

- $u \geqslant 0, v \geqslant 0, u+v \leqslant 1$, in $\bar{\Omega} \times[0, T]$;

- $\quad u$ and $v$ solve the initial value problem (1.2), (1.3), (2.1).

It is quite useful to replace the $v$-equation in (1.3) by an equivalent equation for $p=u+v$ :

$$
p_{t}(\mathbf{x}, t)=\int_{\Omega} k(\mathbf{x}, \mathbf{y}, p(\mathbf{x}, t))[(\gamma-\rho) u(\mathbf{y}, t)-\rho p(\mathbf{y}, t)] \mathrm{d} \mathbf{y}-\alpha[p(\mathbf{x}, t)-u(\mathbf{x}, t)] .
$$

\subsection{A priori estimates and uniqueness}

We begin with a rough a priori estimate.

LEMma 2.1 Under assumptions (A) and (B.1), any solution to (1.2), (1.3), (2.1) is a priori bounded, for bounded $t$.

Proof. Let us define

$$
\|u\|_{t}=\max _{\bar{\Omega} \times[0, t]}|u(\mathbf{x}, \tau)|, \quad\|v\|_{t}=\max _{\bar{\Omega} \times[0, t]}|v(\mathbf{x}, \tau)| .
$$

From (1.2), (2.1), we have

$$
\|u\|_{t} \leqslant \max _{\bar{\Omega}}\left|u_{0}\right|+\delta \gamma \int_{0}^{t}\|u\|_{\tau} \mathrm{d} \tau,
$$

where we used the normalization condition $\int K(x, y) \mathrm{d} y \leqslant 1$. Then from Gronwall's Lemma, we obtain

$$
\|u\|_{t} \leqslant \max _{\bar{\Omega}}\left|u_{0}\right| \mathrm{e}^{\delta \gamma t} \equiv U(t)
$$


Passing to (1.3), we introduce

$$
z(\mathbf{x}, t)=v(\mathbf{x}, t) \mathrm{e}^{\alpha t}
$$

so that

$$
z_{t}(\mathbf{x}, t)=(1-\delta) \gamma F(p) \mathrm{e}^{\alpha t} \int_{\Omega} K(\mathbf{x}, \mathbf{y}) u(\mathbf{y}, t) \mathrm{d} \mathbf{y}+\rho F(p) \int_{\Omega} K(\mathbf{x}, \mathbf{y}) z(\mathbf{y}, t) \mathrm{d} \mathbf{y} .
$$

We obtain the estimate

$$
\left|z_{t}(\mathbf{x}, t)\right| \leqslant(1-\delta) \gamma \mathrm{e}^{\alpha t} U(t)+\rho\|z\|_{t}
$$

and consequently

$$
\|v\|_{t} \leqslant \max _{\bar{\Omega}}\left|v_{0}\right| \mathrm{e}^{(\rho-\alpha) t}+(1-\delta) \gamma \max _{\bar{\Omega}}\left|u_{0}\right| \mathrm{e}^{(\rho+\gamma \delta) t} \equiv V(t) .
$$

In deriving the above estimates we replaced $F(p)$ by 1 , thus obtaining a rough estimate, which is exponentially growing in time. We will show later that $u$ and $v$ are indeed uniformly bounded by 1 .

At this point, we are in a position of proving the following result on continuous dependence on initial conditions:

TheOREM 2.1 Let $u^{(i)}, v^{(i)}$ solve (1.2), (1.3) with data $u_{0}^{(i)}, v_{0}^{(i)}, i=1,2$, and let assumptions (A) and (B.1) be satisfied. Denote by $\Delta u, \Delta v, \Delta p$ the differences $u^{(1)}-u^{(2)}$, etc. Then

$$
\|\Delta u\|_{t}+\|\Delta v\|_{t} \leqslant G \mathrm{e}^{g t} \max _{\bar{\Omega}}\left[\Delta u_{0}+\Delta v_{0}\right],
$$

where $G, g$ are known constants.

Proof. Following the same procedure which we used to get (2.4), we obtain

$$
\|\Delta u\|_{t} \leqslant \mathrm{e}^{\delta \gamma t} \max _{\bar{\Omega}}\left|\Delta u_{0}\right|+U(t) \int_{0}^{t} L\|\Delta p\|_{\tau} \mathrm{d} \tau,
$$

where $L$ is the Lipschitz constant of $F(p)$ and $U(t)$ (see 2.4) is the larger of the upper estimates for $u^{(1)}$ and $u^{(2)}$. Moreover, we get

$$
\left|\Delta z_{t}\right| \leqslant \mathrm{e}^{\alpha t} \gamma(1-\delta)\left\{\|\Delta u\|_{t}+L U(t)\|\Delta p\|_{t}\right\}+\rho\|\Delta z\|_{t}+\rho L V(t) \mathrm{e}^{\alpha t}\|\Delta p\|_{t},
$$

where $z^{(i)}$ are defined according to (2.5). Thus, $\|\Delta z\|_{t}$ can be estimated via Gronwall's Lemma and we get

$$
\|\Delta v\|_{t} \leqslant \mathrm{e}^{\rho t}\left\{\max _{\bar{\Omega}}\left|\Delta v_{0}\right|+\mathrm{e}^{\delta \gamma t} \max _{\bar{\Omega}}\left|\Delta u_{0}\right|\right\}+b_{1} e^{b_{2} t} \int_{0}^{t}\|\Delta p\|_{\tau} \mathrm{d} \tau,
$$

for some known constants $b_{1}, b_{2}$.

Summing (2.9) and (2.10), we get an inequality in terms of $\Delta p$ and using again Gronwall's Lemma we conclude the proof.

As a consequence, we have uniqueness: 
Corollary 2.1 Under assumptions (A) and (B.1), system (1.2), (1.3) with data (2.1) has at most one solution.

Sharper estimates on the bounds of $u$ and $v$ are summarized in the following lemma.

Lemma 2.2 Assume (A), (B.1) are satisfied and let $u, v$ solve equations (1.2), (1.3) in $\Omega \times\left(t_{1}, t_{2}\right)$ for some $0 \leqslant t_{1}<t_{2}$.

(1) If $u\left(\mathbf{x}, t_{1}\right) \geqslant 0, v\left(\mathbf{x}, t_{1}\right) \geqslant 0$ for all $\mathbf{x} \in \Omega$, then

$$
u(\mathbf{x}, t) \geqslant 0, v(\mathbf{x}, t) \geqslant 0, \quad \text { in } \Omega \times\left[t_{1}, t_{2}\right] .
$$

(2) Assume that $1 \geqslant u\left(\mathbf{x}, t_{1}\right) \geqslant 0,1 \geqslant v\left(\mathbf{x}, t_{1}\right) \geqslant 0$ for all $\mathbf{x} \in \Omega$, then

(i) if $u\left(\hat{\mathbf{x}}, t_{1}\right)=p\left(\hat{\mathbf{x}}, t_{1}\right)=1$, for some $\hat{\mathbf{x}} \in \Omega$, then $u(\hat{\mathbf{x}}, t)=1, v(\hat{\mathbf{x}}, t)=0$, for $t \in\left[t_{1}, t_{2}\right]$.

(ii) if $u(\hat{\mathbf{x}}, t)<1$, for some $\hat{\mathbf{x}} \in \Omega$, then $u(\hat{\mathbf{x}}, t)<1$, for $t \in\left[t_{1}, t_{2}\right]$.

(3) If $p\left(\hat{\mathbf{x}}, t_{1}\right)<1$, for some $\hat{\mathbf{x}} \in \Omega$, then $p(\hat{\mathbf{x}}, t)<1$, for $t \in\left(t_{1}, t_{2}\right)$.

(4) If $v\left(\hat{\mathbf{x}}, t_{1}\right)<1$, for some $\hat{\mathbf{x}} \in \Omega$, then $v(\hat{\mathbf{x}}, t)<1$, for $t \in\left(t_{1}, t_{2}\right)$.

Proof.

(1) Let us start by assuming that

$$
u\left(\mathbf{x}, t_{1}\right) \geqslant \varepsilon>0, \quad z\left(\mathbf{x}, t_{1}\right) \geqslant \varepsilon>0, \quad \mathbf{x} \in \Omega,
$$

(where $z$ is defined by (2.5)), for some $\varepsilon \in(0,1)$. Let $\bar{t} \in\left(t_{1}, t_{2}\right)$ be the first time for which there exists an $\overline{\mathbf{x}} \in \bar{\Omega}$ such that $u(\overline{\mathbf{x}}, \bar{t})=0$. But since (1.2) ensures that $u_{t} \geqslant 0$ as long as $u \geqslant 0$, the solution cannot decay below 0 and $u(\mathbf{x}, t) \geqslant 0$. The same argument shows that $z \geqslant 0$ in $\Omega \times$ $\left[t_{1}, t_{2}\right]$, so that $v(\mathbf{x}, t) \geqslant 0$. Letting $\varepsilon$ go to zero and using Theorem 2.1 concludes the proof of part (1).

(2) To show (i), we get from item (1) that $u \geqslant 0$. Hence $u_{t}(\hat{\mathbf{x}}, t) \geqslant 0$ for all $t \in\left(t_{1}, t_{2}\right)$ and consequently $u(\hat{\mathbf{x}}, t) \geqslant 1$, for all $t \in\left(t_{1}, t_{2}\right)$. But since item (1) ensures $v(\hat{\mathbf{x}}, t) \geqslant 0$, we obtain $p(\hat{\mathbf{x}}, t) \geqslant 1$ and then $F(p(\hat{\mathbf{x}}, t))=0$ in $\left(t_{1}, t_{2}\right)$. This implies that $u_{t}=0$ and $u=1$ in the whole interval.

To prove (ii), assume $\bar{t}$ be the first time in $\left(t_{1}, t_{2}\right)$ such that $u(\hat{\mathbf{x}}, \bar{t})=1$. From item (1), we have

$$
v(\mathbf{x}, t) \geqslant 0, \quad 0 \leqslant u(\mathbf{x}, t) \leqslant p(\mathbf{x}, t), \quad \text { in } \Omega \times\left[t_{1}, t_{2}\right],
$$

and hence

$$
0 \leqslant u_{t}(\mathbf{x}, t) \leqslant \delta \gamma F(u(\mathbf{x}, t)), \quad \text { in } \Omega \times\left[t_{1}, t_{2}\right] .
$$

Since $F$ is the Lipschitz continuous and $F(1)=0$, we conclude that the value $u=1$ cannot be reached in a finite time.

(3) We note that the previous results ensure that $u(\hat{\mathbf{x}}, t)<1$, for $t \in\left(t_{1}, t_{2}\right)$. Thus, if $\hat{t}$ is the first time such that $p(\hat{\mathbf{x}}, \hat{t})=1$, we would have $v(\hat{\mathbf{x}}, \hat{t})>0$ and, because of $(2.2), p_{t}(\hat{\mathbf{x}}, \hat{t})=-\alpha v(\hat{\mathbf{x}}, \hat{t})<0$, leading to a contradiction.

(4) A similar argument as used in item (3) gives item (4). 
Now we can apply the above a priori results to any solution of Problem (P) if (A) and (B) are satisfied, just letting $t_{1}=0$ in the previous lemmas. We summarize these facts in the following result on global bounds.

Theorem 2.2 If (A) and (B) are satisfied, then for any solution of Problem (P), we have

(1) The 'saturated' regions (i.e. regions where $p=1$ ) exist only if they exist initially, and never expand.

(2) Saturated regions shrink if and only if they contain non-stem cancers cells.

(3) All solutions of (1.2), (1.3), (2.1) are solutions of Problem (P). Assumption (B) on the data together with the results of this section ensure that $u \geqslant 0, v \geqslant 0, u+v \leqslant 1$, in $\Omega \times[0, T]$.

\subsection{Existence}

Theorem 2.3 Under assumptions (A), (B), Problem (P) has a unique global solution.

Proof. We use a fixed-point argument to show that system (1.2), (1.3), (2.1) has a unique solution $(u, v)$ in a subset of the space $\Lambda_{T}=C(\bar{\Omega} \times[0, T]) \times C(\bar{\Omega} \times[0, T])$ for $T$ small enough. Then the results of the previous section will guarantee that the same pair actually solves Problem (P) (i.e. $u, v$ are in the physical range) and has all the properties shown there (including continuous dependence on the initial data).

Proceeding as in the previous Section, we already know that, if we prescribe $p(\mathbf{x}, t)$ in (1.2), (1.3), the possible solutions of the corresponding system satisfy the a priori bounds

$$
\begin{aligned}
& \|u\|_{t} \leqslant\left(\max _{\bar{\Omega}} u_{0}\right) \mathrm{e}^{\delta \gamma t} \equiv U(t) . \\
& \|v\|_{t} \leqslant \frac{1-\delta \gamma}{\alpha+\delta \gamma}\left(\max _{\bar{\Omega}} u_{0}\right)\left[\mathrm{e}^{\delta \gamma t}-\mathrm{e}^{-\alpha t}\right] \mathrm{e}^{\rho t}+\left(\max _{\bar{\Omega}} v_{0}\right) \mathrm{e}^{(\rho-\alpha) t} \equiv V(t) .
\end{aligned}
$$

Thus, we can take a pair $(u, v) \in \Lambda_{T}$ such that $u(\mathbf{x}, 0)=u_{0}(\mathbf{x}), v(\mathbf{x}, 0)=v_{0}(\mathbf{x})$, and respecting the bounds (2.13), (2.14). We denote such a subset by $\Lambda_{T}^{0}$. We use $p=u+v$ in (1.2), (1.3) and we look for a solution $(\tilde{u}, \tilde{v})$ of

$$
\begin{aligned}
& \tilde{u}_{t}(\mathbf{x}, t)=\delta \gamma F(p(\mathbf{x}, t)) \int_{\Omega} K(\mathbf{x}, \mathbf{y}) \tilde{u}(\mathbf{y}, t) \mathrm{d} \mathbf{y} \\
& \tilde{v}_{t}(\mathbf{x}, t)=(1-\delta) \gamma F(p(\mathbf{x}, t)) \int_{\Omega} K(\mathbf{x}, \mathbf{y}) \tilde{u}(\mathbf{y}, t) \mathrm{d} \mathbf{y}+\rho F(p(\mathbf{x}, t)) \int_{\Omega} K(\mathbf{x}, \mathbf{y}) \tilde{v}(\mathbf{y}, t) \mathrm{d} \mathbf{y}-\alpha \tilde{v}(\mathbf{x}, t),
\end{aligned}
$$

in the same space $\Lambda_{T}^{0}$.

Since (2.16) is now a linear equation, the existence of the pair $(\tilde{u}, \tilde{v})$ is easily shown using a simple fixed-point argument. All we have to do is to analyse the differences $\Delta \tilde{u}=\tilde{u}^{(1)}-\tilde{u}^{(2)}, \Delta \tilde{v}=\tilde{v}^{(1)}-\tilde{v}^{(2)}$ corresponding to the pairs $\left(\tilde{u}^{(1)}-\tilde{u}^{(2)}\right),\left(\tilde{v}^{(1)}-\tilde{v}^{(2)}\right)$ generating the sums $p^{(1)}, p^{(2)}$. 
We have that $\|\Delta \tilde{u}\|_{t},\|\Delta \tilde{v}\|_{t}$ satisfy a system of Gronwall type inequalities with free terms of the form

$$
\int_{0}^{t}\left(\|\Delta u\|_{\tau}+\|\Delta v\|_{\tau}\right) \Gamma(\tau) \mathrm{d} \tau,
$$

where $\Gamma(\tau)$ is either $U(\tau)$ or $V(\tau)$ times some constant. These are the only terms including $\|\Delta u\|_{t}$ and $\|\Delta v\|_{t}$. Then we can select $T$ such that the mapping $(u, v) \rightarrow(\tilde{u}, \tilde{v})$ is a contraction in $\Lambda_{T}^{0}$.

Once we have proved existence for sufficiently small $T$, we can say that $u(\mathbf{x}, T), v(\mathbf{x}, T)$ satisfy the same assumptions as $u_{0}(\mathbf{x}), v_{0}(\mathbf{x})$. The nonlinearity $F$ and the kernel $K$ are bounded, therefore the argument can be iterated, yielding global in time existence.

\section{Numerical examples}

We use numerical simulations of the iDEs (1.2), (1.3) to illustrate two effects. First, we show that this model shows the tumour growth paradox. A larger death rate of CC will eventually lead to a larger tumour. This effect was already found in the in silico-model of Enderling et al. (2009) and in the ODE model of Hillen et al. (2013). Here we confirm that this effect also exists in the integro-differential formulation. We also find that the tumour with larger death rate is stem cell rich, in contrast to the case of a low death rate. We investigate the sensitivity of the tumour growth paradox on the model parameters. We find that this paradox exists for all considered parameter combinations, however, in many cases it is not very pronounced.

Secondly, we study one illustrative example of an incomplete radiation treatment. After a rather short time, we see that the treated tumour grows larger than it would have been without treatment. Both observations reinforce the importance of the stem cell compartment and its increased resistance to treatment modalities.

Equations (1.2) and (1.3) are solved for $x \in[-L, L]$, and $t \in[0,200]$. We choose a standard linear volume filling term (Painter \& Hillen, 2002)

$$
F(p)=(1-p)^{+}=\max (0,1-p)
$$

and Gaussian redistribution kernels

$$
K_{u}(x, y)=\frac{1}{\sigma_{u} \sqrt{\pi}} \mathrm{e}^{-(x-y)^{2} / \sigma_{u}^{2}}, \quad K_{v}(x, y)=\frac{1}{\sigma_{v} \sqrt{\pi}} \mathrm{e}^{-(x-y)^{2} / \sigma_{v}^{2}},
$$

where $\sigma_{u}, \sigma_{v}$ are the standard deviations. To solve system (1.2), (1.3), we use a finite difference scheme, with an explicit forward method in time. The integrals appearing in the equations are computed by the trapezoidal rule.

The initial data are chosen to describe a concentrated tumour mass in the centre of the domain which consists of stem cells only:

$$
u_{0}(x)=\mathrm{e}^{-10 x^{2}} \quad \text { and } \quad v_{0}(x)=0 .
$$

The following parameter values define our standard parameter set

$$
\delta=0.2, \quad \sigma_{u}=0.5, \quad \sigma_{v}=0.1, \quad \gamma=1.0, \quad \rho=0.5
$$

We vary the death rate $\alpha$ between two representative values, a small value of $\alpha=0.2$ and a large value of $\alpha=2$. 

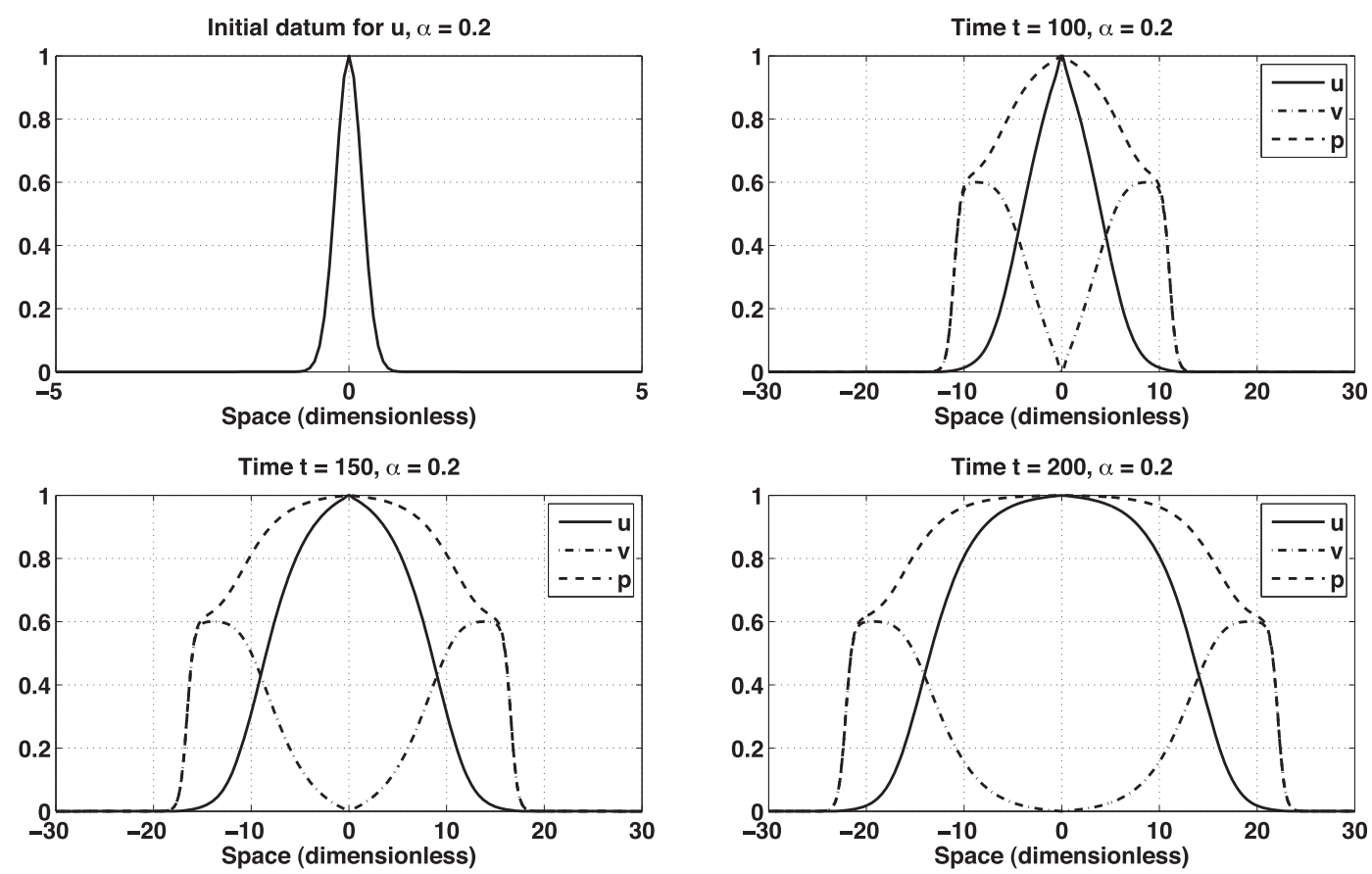

FIG. 1. Plot of $u, v, p$ at selected time instants, with initial conditions (3.3) and parameters from (3.4). Case $\alpha=0.2$. Detail of space interval $[-30,30]$.

The following examples show the sensitivity of the system to the mortality rate $\alpha$ and, in particular, the possible appearance of the tumour growth paradox after a suitable time.

\subsection{Evidence of the tumour growth paradox}

The distribution of $u, v, p$ at selected times are reported in Fig. 1 for $\alpha=0.2$ and in Fig. 2 for $\alpha=2$. For small $\alpha$ value ( $\alpha=0.2$ in Fig. 1 ), we see that the invasion wave is dominated by CC, whereas in the centre the stem cell population establishes dominance. This behaviour is quite different for large $\alpha=2$ (Fig. 2), where the invasion is clearly stem cell dominated and the non-stem CCs play only a minor role at the invasion front. To elucidate the appearance of the tumour paradox, we consider the total population of tumour cells, namely

$$
\Pi(t)=\int_{-L}^{L} p(x, t) \mathrm{d} x
$$

and look for its time evolution, in dependence on $\alpha$ (Fig. 3, left). The occurrence of the tumour growth paradox is evident around $t=60$. The population with a lower death rate $\alpha$ initially grows faster, but eventually is exceeded by the populations with the higher $\alpha$ value.

To show the tumour growth paradox from a different point of view, the evolution of points tracking a fixed value of $p$ was also computed. In Fig. 3, right, the $80 \%$ level set of $p$ is shown

$$
x_{[p=0.8]}(t)=\left\{x^{*} \in[0, L]: p\left(x^{*}, t\right)=0.8\right\}
$$



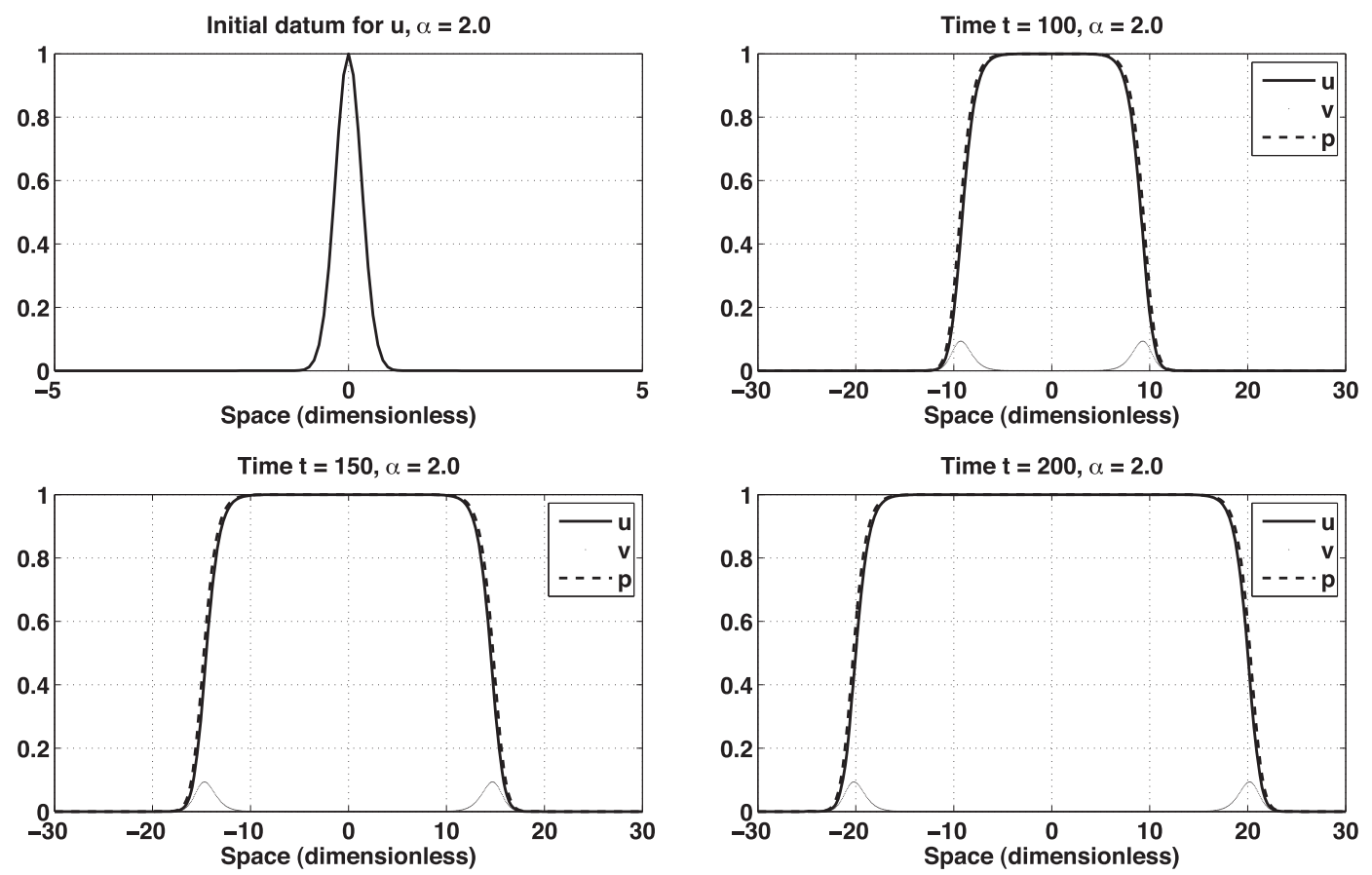

FIG. 2. Plot of $u, v, p$ at selected time instants, with initial conditions (3.3) and parameters from (3.4). Case $\alpha=2.0$. Detail of space interval $[-30,30]$.
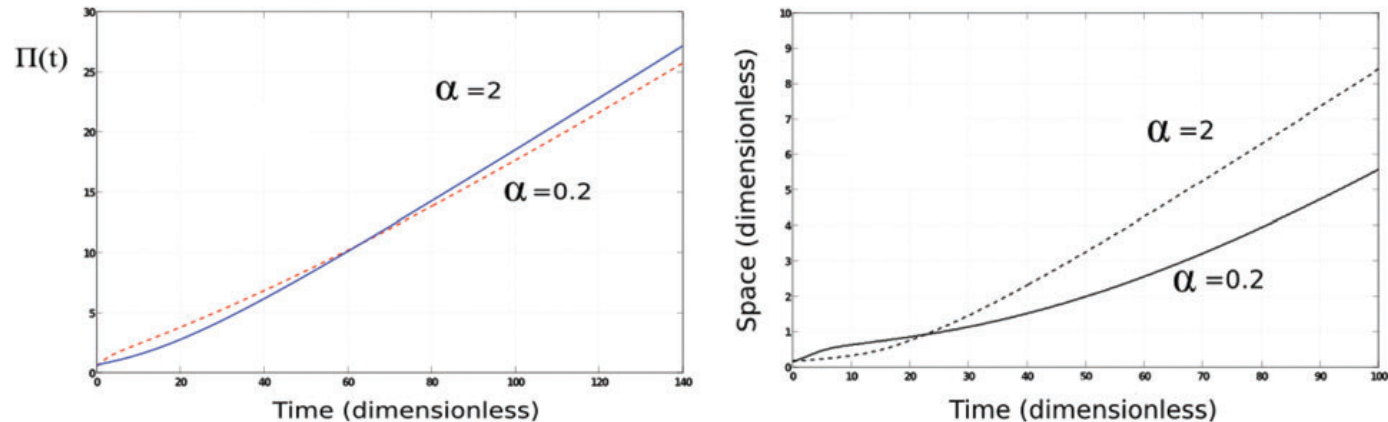

FIG. 3. Left: Time evolution of total population, $\Pi(t)$, for $\alpha=0.2$ and $\alpha=2.0$. Right: Time evolution of $x_{[p=0.8]}(t)$, for $\alpha=0.2$ and $\alpha=2.0$.

for $\alpha=0.2$ and $\alpha=2$. The slope of the corresponding curves is a measure for the invasion speed. Clearly, the curve for larger $\alpha$ has a larger invasion speed. We plan to use a forthcoming paper to study the invasion travelling wave speeds in detail.

\subsection{Dependence on model parameters}

To investigate how the tumour growth paradox depends on the model parameters, we investigate a whole range of parameter sets. We found that a faster spread of the stem cells, expressed by an increased variance $\sigma_{u}$, increases the tumour spread, but decreases the tumour growth paradox. By a decreased 

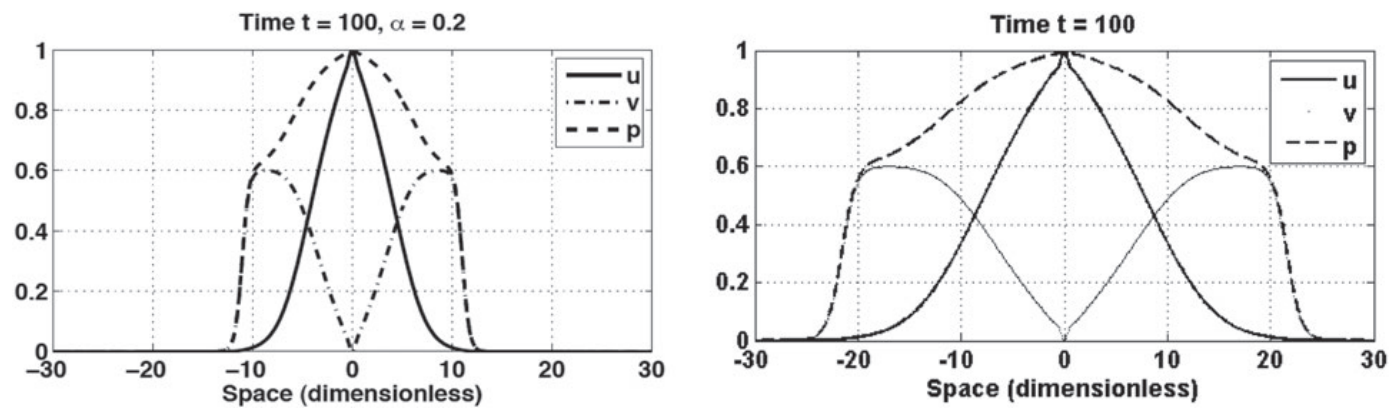

FIG. 4. Simulations as in Fig. 1 at $t=100$. Here $\alpha=0.2$ and $\sigma_{u}=0.5$ (left) and $\sigma_{u}=1$ (right). All other parameters are as in (3.4).
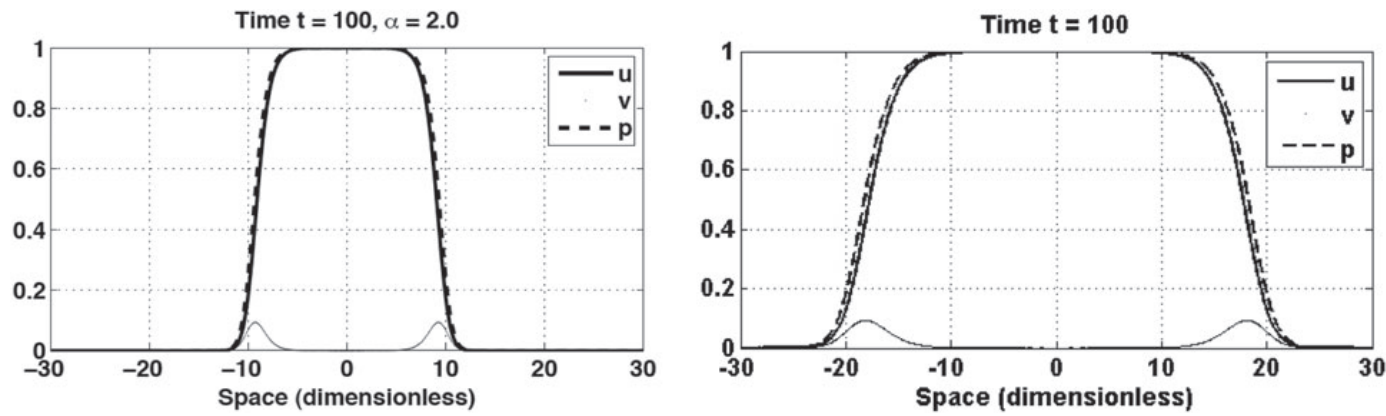

FIG. 5. Simulations as in Fig. 1 at $t=100$. Here $\alpha=2$ and $\sigma_{u}=0.5$ (left) and $\sigma_{u}=1$ (right). All other parameters are as in (3.4).
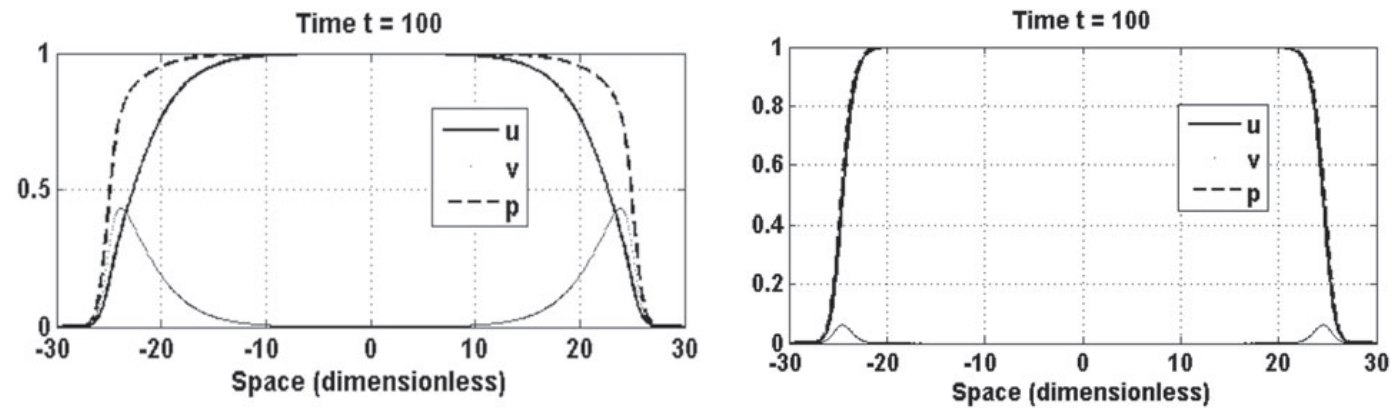

FIG. 6. Simulations as in Fig. 1 at $t=100$. Here $\delta=0.5$ and $\alpha=0.2$ (left) and $\alpha=2$ (right). All other parameters are as in (3.4).

tumour growth paradox we mean that the difference in population sizes between $\alpha=0.2$ and $\alpha=2$ is reduced when compared with models with smaller $\sigma_{u}$. In Fig. 4, we compare the simulations at time $t=100$ for $\alpha=0.2$ and $\sigma_{u}=0.5$ (left) and $\sigma_{u}=1$ (right). The spread in Fig. 4 (right) is faster than the spread on the left. Still, the invasion is CC dominated. In the case of $\alpha=2$, we also observe increased invasion speeds for $\sigma_{u}=1$, but in this case the invasion is CSC dominated (see Fig. 5).

A variation of the spread rate for $v, \sigma_{v}$, only has a minimal effect on the simulation results (not shown). However, a variation of the rate of CSC renewal $\delta$ makes a big difference. In Fig. 6 , we 

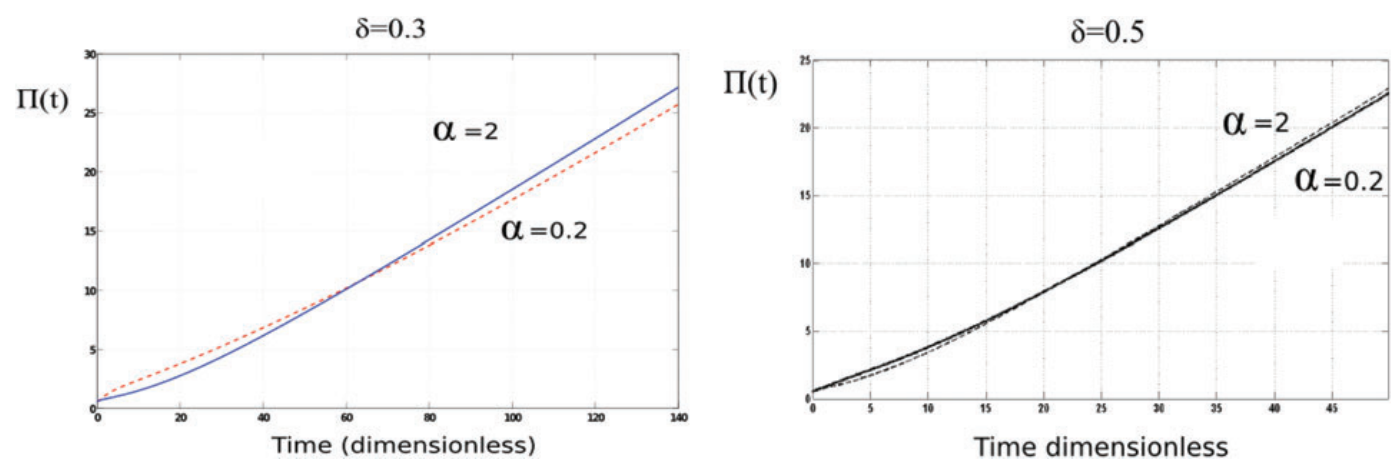

FIG. 7. Time evolution of the total population $\Pi(t)$ for $\delta=0.3$ (left) and $\delta=0.5$ (right). All other parameters are as in (3.4).

increase $\delta$ from 0.2 to 0.5 . We show the simulation for $t=100$ and $\alpha=0.2$ on the left and $\alpha=2$ on the right. In both cases, the invasion is driven by the CSC compartment. The tumour growth paradox still arises, as seen in Fig. 7 (right), but the difference in the total populations is negligible for $\delta=0.5$.

\subsection{Simulating the effect of a radiation treatment}

To illustrate the basic effect of stem cell resistance to radiation, we use a rather ad hoc approach. We consider one radiation dose (at time $t^{*}=50$ in this example), and we denote the reduction of the cell population by a factor $1-\phi, \phi \in[0,1]$. We are aware of detailed radiation treatment models involving the linear quadratic model and tumour control probabilities, but a detailed discussion of this framework does not add to the argument we want to make here. For details on radiation treatment modelling, we refer the reader to Gong et al. (2011) and Dawson \& Hillen (2006). Here, at the treatment time $t^{*}$, we choose new initial data as

$$
\begin{aligned}
& u\left(x, t^{*}\right)=(1-\theta \phi) u\left(x, t^{*}-\right), \\
& v\left(x, t^{*}\right)=(1-\phi) v^{*}\left(x, t^{*}-\right),
\end{aligned}
$$

where $t^{*}-=\lim _{t \rightarrow t^{*}, t<t^{*}}$. The term $(1-\phi)$ denotes the surviving fraction of CC while $(1-\theta \phi)$ denotes the larger surviving fraction of CSC. The value of $\theta, 0 \leqslant \theta \leqslant 1$, describes radio resistance of stem cells.

Results reported in Fig. 8 correspond to $\phi=0.95, \theta=0$, for $\alpha=0.2$, as selected times after treatment. Other model parameters have value as in (3.4). The upper figure shows the effect of radiation at $t=50$. The solid line shows the tumour distribution $p(x, t)$ just before treatment, and the dashed line just after treatment. The reduction is lowest in the centre, where most CSC are residing. The lower figure in Fig. 8 shows the tumour evolution at time $t=150$, where the solid line (without treatment) basically coincides with the dashed line (with treatment). Hence incomplete treatment seems to have no effect on the tumour.

In Fig. 9, we show the total population on the left and the level set $p=0.8$ on the right. Here we see that the progression with treatment (dashed line) is slightly faster than without treatment (solid line). Again, a mild tumour growth paradox is at play. 

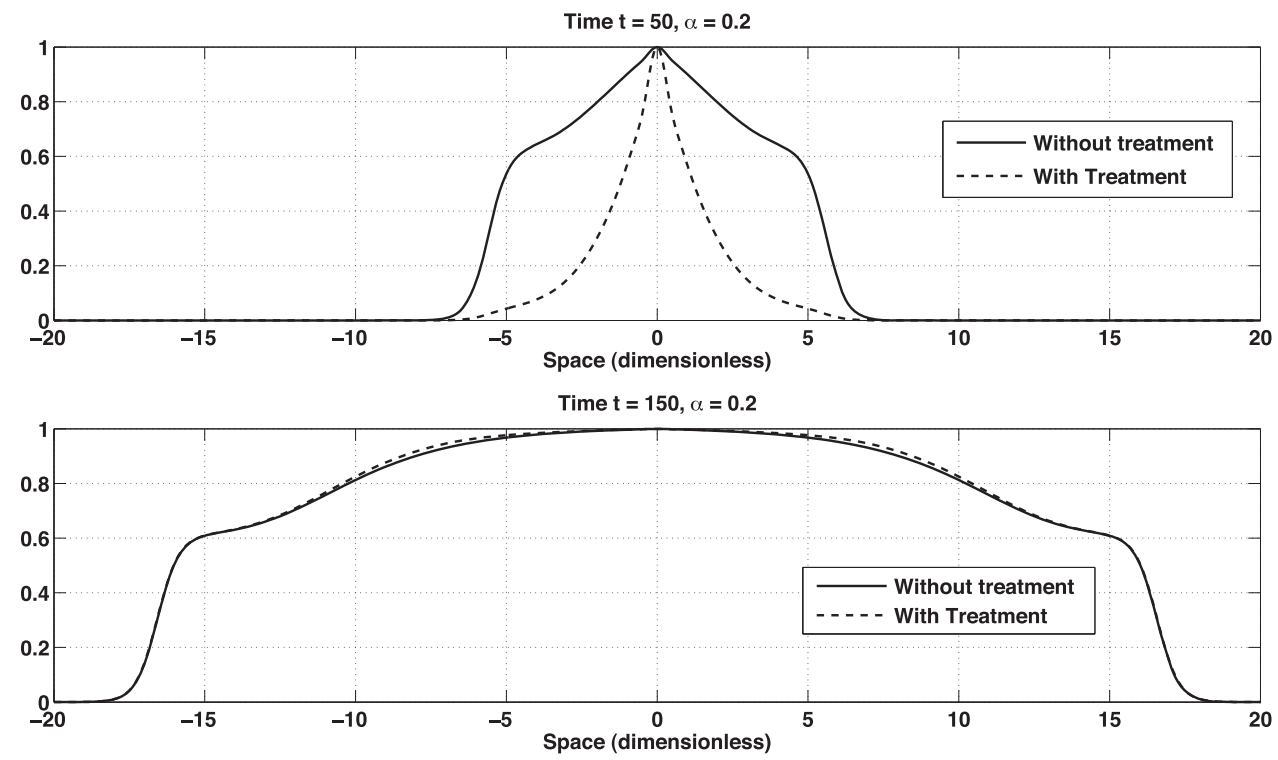

FIG. 8. Plot of $p$ at selected time instants in case of radiation treatment at $t=50$. Case $\alpha=0.2$. Detail of space interval [-20,20].
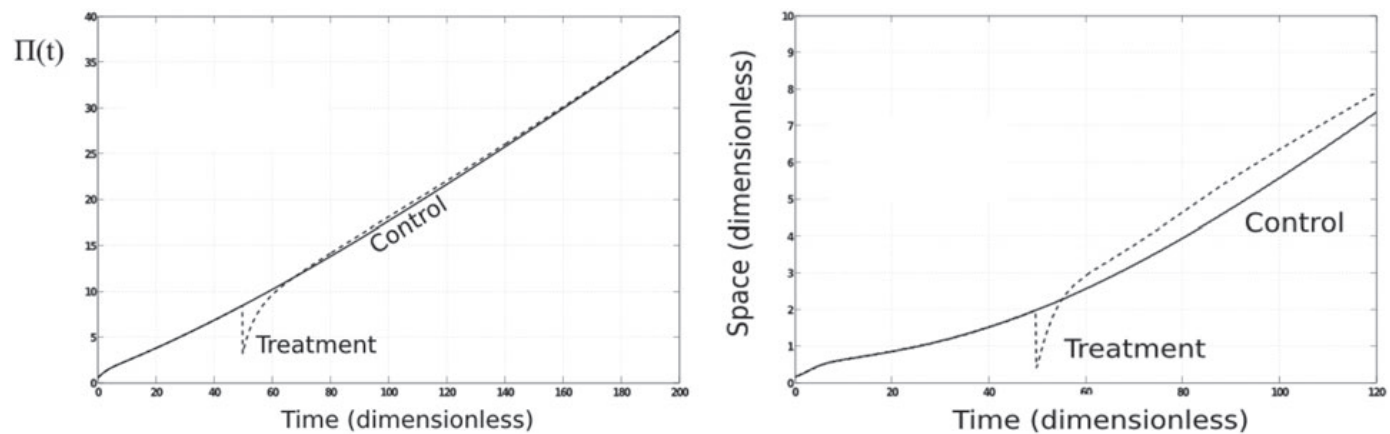

FIG. 9. Left: Time evolution of total population, $\Pi(t)$, for $\phi=0.95$ and $\alpha=0.2$. Right: Time evolution of $x_{[p=0.8]}(t)$, for $\phi=0.95$ and $\alpha=0.2$.

\section{Discussion}

The increased resistance of CSCs to various treatment modalities, when compared with non-stem CC, is one of the major obstacles of cancer treatment. CSCs have been used to explain many observed phenomena of cancer progression, including the tumour growth paradox. Our model extends previous results of the tumour growth paradox. The individual-based model of Enderling et al. (2009), the ODE of Hillen et al. (2013) and our system (1.2), (1.3) make it perfectly clear, that, as soon as CSC and CC compete for space and resources, a tumour growth paradox exists.

We have studied an ad hoc radiation treatment with a single application of radiation (one fraction) and we found that incomplete treatment leads to a selection of CSC. We could include more realistic treatments, or chemotherapy and other treatments, but the principle will be the same. Any stress applied 
to the tumour will lead to a selection of CSC, and this is the point we wanted to make here. The consideration of more realistic treatments is left to further studies. It is clear, however, that a successful treatment needs to target CSC. One such strategy was suggested by Youssefpour et al. (2012). They suggest that conventional treatment, such as radiation treatment, should be combined with a differentiation therapy. Differentiation therapy describes a chemotherapy, where the chemotherapeutic agent pushes stem cells into the differentiation cascade. Several candidate drugs are known and currently tested. In Bachman \& Hillen (2012), the above ODE stem cell model (1.1) was used to test Lowengrub's hypothesis for specific cancers (secondary brain tumours, breast cancer and head and neck cancer). It was confirmed that for brain and head and neck cancers a combination therapy can reduce the necessary radiation dose, while the benefit for breast cancer treatment was limited (according to the mathematical model).

As outlined before, our CSC model (1.2), (1.3) falls into the class of birth-jump models as introduced in Hillen et al. (2014). The existence theory developed here is only a start. Many more interesting mathematical questions lie ahead for future research, including models for invasion, travelling waves and spatial pattern formation.

\section{Acknowledgements}

We are grateful to detailed comments from two anonymous referees.

\section{Funding}

T.H. is supported through NSERC.

\section{REFERENCES}

Bachman, J. \& Hillen, T. (2012) Mathematical optimization of the combination of radiation and differentiation therapies of cancer. Front. Mol. Cell. Oncol. doi: 10.3389/fonc.2013.00052.

Beretta, E., Capasso, V. \& Morozova, N. (2012) Mathematical modelling of cancer stem cells population behavior. Math. Model. Nat. Phenom., 7, 279-305.

Dawson, A. \& Hillen, T. (2006) Derivation of the tumour control probability (TCP) for a cell cycle model. Comput. Math. Methods Med., 7, 121-142.

Dingli, D. \& Michor, F. (2006) Successful therapy must eradicate cancer stem cells. Stem Cells, 24, 2603-2610.

DitTMAR, T. \& Zänker, K. S. (2013) Role of Cancer Stem Cells in Cancer Biology and Therapy. Boca Raton, FL, USA: CRC Press.

Enderling, H., Anderson, A. R. A., Chaplain, M. A. J., Beheshti, A., Hlatky, L. \& Hahnfeldt, P. (2009) Paradoxical dependencies of tumour dormancy and progression on basic cell kinetics. Cancer Res., 69, 8814-8821.

Ganguli, R. \& Puri, I. K. (2006) Mathematical model for the cancer stem cell hypothesis. Cell Prolif., 39, 3-14.

Gong, J., dos Santos, M. M., Finlay, C. \& Hillen, T. (2011) Are more complicated tumour control probability models better? Math. Med. Biol., 30, 1-19.

Hanahan, D. \& Weinberg, R. A. (2011) Hallmarks of cancer: the next generation. Cell, 144, 646-674.

HeK, G. (2010) Geometric singular perturbation theory in biological practice. J. Math. Biol., 60, 347-386.

Hillen, T., Enderling, H. \& Hahnfeldt, P. (2013) The tumour growth paradox and immune system-mediated selection for cancer stem cells. Bull. Math. Biol., 75, 161-184.

Hillen, T., Greese, B., Martin, J. \& De Vries, G. (2014) Birth-jump processes. J. Biol. Dyn. doi: $10.1080 / 17513758.2014 .950184$.

Maddalena, L. (2014) Analysis of an integro-differential system modelling tumour growth. Appl. Math. Comp., 245, 152-157. 
Painter, K. J. \& Hillen, T. (2002) Volume-filling and quorum-sensing in models for chemosensitive movement. Can. Appl. Math. Quart., 10, 501-543.

Sole, R. V., Rodriguez-Caso, C., Diesboeck, T. S. \& Saldance, J. (2008) Cancer stem cells as the engine of unstable tumour progression. J. Theor. Biol., 253, 629-637.

Stiehl, T. \& Marciniak-Czochra, A. (2012) Mathematical modeling of leukemogenesis and cancer stem cell dynamics. Math. Model. Nat. Phenom., 7, 166-202.

Sun, Z. \& Komarova, N. L. (2012) Stochastic control of proliferation and differentiation in stem cell dynamics. Math. Biosci., 240, 231-240.

Youssefpour, H., Li, X., Lander, A. D. \& Lowengrub, J. S. (2012) Multispecies model of cell lineages and feedback control in solid tumours. J. Theor. Biol., 304, 39-59. 